\title{
Cerebral Autosomal Dominant Arteriopathy with Subcortical Infarcts and Leukoencephalopathy (CADASIL) Syndrome: A Case Report
}

\author{
Suprakash Chaudhury ${ }^{1}$, Rakesh Kumar Singh*2, Samiksh Sahu ${ }^{1}$ and Adnan Kadiyani ${ }^{1}$ \\ ${ }^{1}$ Department of Psychiatry, Dr D Y Patil Medical College, India \\ ${ }^{2}$ Department of Psychiatry, Hind institute of Medical Science, India
}

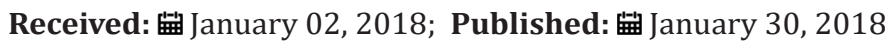

*Corresponding author: Rakesh Kumar Singh, Department of Psychiatry, Hind institute of Medical Science, India

\begin{abstract}
Cerebral autosomal dominant arteriopathy with sub cortical infarcts and leukoencephalopathy (CADASIL) is a rare autosomal dominant genetic disease. A35 year old women presented with progressive loss of memory left sided weakness and hemiplegic and non insulin dependent diabetes mellitus with characteristic MRI findings is reported due to its rarity.
\end{abstract}

Keywords: CADASIL Small vessel disease; Cognitive impairment

\section{Introduction}

Cerebral small vessel disease (SVD) is increasingly being recognized as the cause of stroke, impaired cognitive function and mood disorders in geriatric age group. Commonly SVD is sporadic due to old age and high blood pressure, but occasionally SVD has a monogenic cause. The best known among these is cerebral autosomal dominant arteriopathy with sub cortical infarcts and leuko encephalopathy(CADASIL), a rare type of autosomal dominant cerebral angiopathy. It causes recurrent sub cortical ischemic events and vascular dementia which appear as diffuse white matter abnormality on neuro imaging. It involves mainly the small cerebral vessels; histopathology reveals non-atherosclerotic and non-amyloidal angiopathy. [1] The 1st case of aforementioned symptoms was reported 30 years ago in a Swedish family [2] and the term CADASIL was coined in early 1990's [3] Since then CADASIL has been diagnosed in number of families and ethnic groups all over the world.

The prevalence of the condition varies between 2 and 5 in 100,000 . Clinically the condition manifests in early or middle adulthood with variety of complaints ranging from migraine, with or without aura, which occur in $30-40 \%$ individual, mood disorders, usually depression seen in $30 \%$ people, along with recurrent ischemic events, transient or permanent, accompanied by progressive cognitive impairment leading to dementia and premature death [4-5].

\section{Case Report}

This 35 years old married female patient was brought to the psychiatry outpatient department by her family members with the complaint of gradual decline of memory, difficulty in walking due to residual left sided motor weakness and co morbid Non Insulin dependent Diabetes Mellitus. There was no history of hypertension, depression or psychosis or impaired pain sensation. No history of seizures. EEG done earlier did not reveal any abnormality. There was past history of two episodes of CVA in last 5 years. Family history was not significant for headache but there was history of premature death of uncles. Physical examination showed thin built poorly nourished lady. There was no pallor, cyanosis, icterus, edema or generalized lymphadenopathy. Systemic examination of central nervous system revealed grade 3 motor weakness in left upper and lower limbs. Cranial nerves and fundi were normal. There were no sensory deficits or cerebellar signs. Examination of other systems was normal. Score on Mini Mental Status Examination was 12 out of 20. Relevant investigations including hemoglobin, blood sugar, HbA1c, liver function tests, SGOT, SGPT, alkaline phosphatase, blood urea, serum creatinine, were within normal limits. CT Scan brain 
showed a lacunar infarct in left external capsular region and age inappropriate diffuse cerebral atrophy. MRI of brain revealed diffuse cerebral atrophy with prominent sulcal spaces with periventricular and subcortical arteriosclerotic white matter changes. Small areas of acute to sub acute infarct in rest of the bilateral parietal and frontal lobes support diagnosis of CADASIL Syndrome.

\section{Discussion}

CADASIL gene was first identified on the chromosome 19 in the year 1996 [6] Subsequent studies revealed the mutation of NOTCH3 gene on chromosome 19q12. This gene codes for transmembrane receptor protein which is located on surface of smooth muscle cell which surrounds arteries. When pathological NOTCH3 receptor protein got accumulated in cerebral arteries it resulted in varied symptoms of CADAIL [7].

Patient with CADASIL can present with a variety of psychiatric manifestation in $20-41 \%$ of cases. The psychiatric manifestations range from agoraphobia, psychoses, personality disorders, alcohol and substance use disorders, adjustment disorder to episodic mood disturbances and bipolar affective disorder $[4,8]$.

CADASIL should be suspected in individual with history of transient ischemic attacks, migraine, severe mood disturbances or late onset affective disorder with headache and neurological symptoms [8-9]. MRI studies shows hyper-intense lesion and ischemic lesion in Basal Ganglia and frontal location of white matter is associated with mood disturbances in patient of CADASIL [10-11].

To conclude it is advisable to do MRI brain in patients presenting with late onset Psychiatric Disturbances and a diagnosis of CADASIL should be considered as a possible differential diagnosis whenever a marked leukoencephalopathy is reported [12].

\section{References}

1. VikelisaM, XifarasbM, MitsikostasaD (2006) CADASIL: a short review of the literature and a Description of the first family from Greece. Functional Neurology 21(2): 77-82.
2. Sourander P, Walinder J (1977) Hereditary multi-infarct dementia. Morphological and clinical studies of a new disease. Acta Neuropathol 39(3): 247-254.

3. Tournier Lasserve E, Joutel A, Melki J, Weissenbach J, Lathrop GM, et al. (1993) Cerebral autosomal dominant arteriopathy with sub cortical infarcts and leukoencephalopathy maps to chromosome 19q12. Nat Genet 3(3): 256-259.

4. Di DonatoI, BianchiS, De Stefano N, Dichgans M, Dotti MT, et al. (2017) Cerebral Autosomal Dominant Arteriopathy with Subcortical Infarcts and Leukoencephalopathy (CADASIL) as a model of small vessel disease: update on clinical, diagnostic, and management aspects. BMC Med 15(1): pp 41.

5. AdibSamiiP, Brice G, Martin RJ, Markus HS (2010) Clinical spectrum of CADASIL and the effect of cardiovascular risk factors on phenotype: study in 200 consecutively recruited individuals. Stroke 41(4): 630-640.

6. JoutelA, CorpechotC, Ducros A, Vahedi K, Chabriat H et al. (1996) Notch3 mutations in CADASIL, a hereditary adult-onset condition causing stroke and dementia. Nature 383(6602): 707-710.

7. Meschia JF, Brott TG, Brown RD (2005) genetics of cerebrovascular disorders. Mayo Clin Proc 80(1):122-132.

8. VerïnM, RollandY, LandgrafF, ChabriatH, Bompais B, et al. (1995) new phenotype of the cerebral autosomal dominant arteriopathy mapped to chromosome 19: migraine as the prominent clinical feature. J Neurol Neurosurg Psychiatry 59(6): 579-585.

9. Kumar SK, Mahr G (1997) CADASIL presenting as bipolar disorder. Psychosomatics 38(4): 397-398.

10. Aylward ED, Roberts Willie JV, Barta PE, Kumar AJ, Harris GJ, et al. (1994) Basal ganglia volume and white matter hyper intensities in patients with bipolar disorder. Am J Psychiatry (151)5: 687-693.

11. Bhatia K, Marsden C (1994) the behavioral and motor consequences of focal lesions of the basal ganglia in man. Brain 117(4): 859-876.

12. LeyheT, WiendlH, BuchkremerG, Wormstall H (2005) CADASIL: under diagnosed in psychiatric patients? Acta Psychiatr Scand 111(5): 392397.

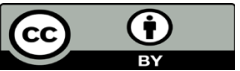

This work is licensed under Creative Commons Attribution 4.0 License

To Submit Your Article Click Here:

Submit Article

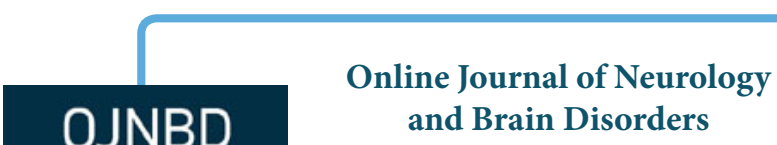
and Brain Disorders

\section{Assets of Publishing with us}

- Global archiving of articles

- Immediate, unrestricted online access

- Rigorous Peer Review Process

- Authors Retain Copyrights

- Unique DOI for all articles 